\title{
High-Temperature Microfluidic Synthesis of CdSe Nanocrystals in Nanoliter Droplets
}

\author{
Emory M. Chan ${ }^{*}$ A. Paul Alivisatos, ${ }^{+*}$ and Richard A. Mathies*"
}

Department of Chemistry, University of California, Berkeley, and Materials Science Division, Lawrence Berkeley National Laboratory, Berkeley, CA 94720

\footnotetext{
* To whom correspondence should be addressed. Email: rich@zinc.cchem.berkeley.edu

${ }^{\dagger}$ Dept. of Chemistry, UC-Berkeley

\$aterials Science Division, Lawrence Berkeley National Laboratory
}

First Submitted: March 4, 2005

Revised: June 8, 2005 


\begin{abstract}
The high-temperature synthesis of CdSe nanocrystals in nanoliter-volume droplets flowing in a perfluorinated carrier fluid through a microfabricated reactor is presented. A flow-focusing nanojet structure with a step increase in channel height reproducibly generated octadecene droplets in Fomblin Y 06/6 perfluorinated polyether at capillary numbers up to 0.81 and with a droplet:carrier fluid viscosity ratio of 0.035 . Cadmium and selenium precursors flowing in octadecene droplets through a hightemperature $\left(240-300^{\circ} \mathrm{C}\right)$ glass microreactor produced high quality CdSe nanocrystals, as verified by optical spectroscopy and transmission electron microscopy. Isolating the reaction solution in droplets prevented particle deposition and hydrodynamic dispersion, allowing the reproducible synthesis of nanocrystals at three different temperatures and four different residence times in the span of four hours. Our synthesis of a wide range of nanocrystals at high temperatures, high capillary numbers, and low viscosity ratio illustrates the general utility of droplet-based microfluidic reactors to encapsulate nanoliter volumes of organic or aqueous solutions and to precisely control chemical or biochemical reactions.
\end{abstract}




\section{Introduction}

The compartmentalization of reagents in nanoliter and sub-nanoliter volumes is valuable for enhancing and controlling chemical reactions. ${ }^{1}$ Reactions in emulsions suspended in bulk fluid, however, are limited by dynamic exchange, phase diagram constraints, and by the inability to manipulate and monitor the individual nanoreactors. Microfabricated devices are now being used to perform reactions in microliter to nanoliter volumes, demonstrating enhanced reaction yield, selectivity, and kinetics. ${ }^{2-6}$ Microfluidics have also been used to generate and control stable, isolated droplets and emulsions $^{7-9}$ for biological analysis, ${ }^{10}$ crystallization, ${ }^{11}$ and chemical synthesis. ${ }^{5,}{ }^{12}$ These systems commonly use water, mild temperatures, and polymeric substrates. Many reactions, however, such as the inorganic nanoparticle synthesis considered here, require organic solvents, elevated temperatures, and air- and water-sensitive reagents. A more general and robust method for performing reactions in nanoliter volumes under challenging conditions must be developed.

Continuous flow microreactors have been used to rapidly grow and characterize inorganic nanocrystals. ${ }^{13-19}$ The synthesis of semiconductor nanocrystals in microfluidic systems provides a stringent test for advanced microfluidic techniques, because such reactions are extremely sensitive to synthetic conditions, involve high temperatures, caustic precursors, and rapid kinetics, and because these reactions produce products that are continuously distributed in size. ${ }^{15}$ In continuous flow microreactors, the particle size distribution is further broadened by the velocity and residence time distributions inherent to pressure-driven flow. ${ }^{15,20}$ Particles can also nucleate and deposit on channel walls, leading to runaway growth, clogging, and unstable reactor conditions, particularly at high temperatures and in small channels. ${ }^{12,17}$

Segmented-flow microfluidics present a potential remedy for the dispersion and deposition challenges inherent to nanoparticle synthesis in microfluidic systems. Gas bubbles introduced in microreactor flows can reduce nanoparticle size distribution. ${ }^{16,21,22}$ Gases, however, change volume 
significantly with pressure and temperature. ${ }^{22}$ Moreover, liquid slugs in such systems still physically contact reactor walls and neighboring slugs, allowing for particle deposition and dispersion. ${ }^{23}$ Alternatively, the encapsulation of precursor solutions in nanoliter-scale droplets flowing in a carrier fluid physically and temporally isolates reactants so that they do not interact with channel walls and so that they can be transported without cross-contamination. ${ }^{24}$ Such droplets can be generated in microfluidic devices by shearing a stream of the droplet phase with the flow of the continuous phase via cross-flow $^{8,25}$ or via flow-focusing ${ }^{9,26}$ geometries. While droplet-based microfluidics have been used to synthesize nanoparticles at room temperature in aqueous solutions, ${ }^{12,27}$ these systems are not compatible with the pyrolytic synthesis of high quality semiconductor nanocrystals. For high-temperature synthesis, the droplet and carrier fluids must be stable, non-interacting, non-volatile, liquid, and immiscible from ambient to reaction temperatures $\left(\sim 300^{\circ} \mathrm{C}\right)$. Octadecene (ODE) has been used as a non-coordinating solvent for high-temperature semiconductor nanocrystal synthesis in bulk fluids, ${ }^{28,}{ }^{29}$ making it a suitable droplet phase. Long-chain perfluorinated polyethers (PFPE's) are liquid, essentially inert, and immiscible with nearly all non-fluorous solvents under nanocrystal synthesis conditions, making them ideal carrier fluids.

To synthesize nanocrystals in droplets at high temperatures in this system, droplets must be generated despite the low interfacial tensions $(5-25 \mathrm{mN} / \mathrm{m})$ of the organic/fluorous solvent pair and the large viscosities $(>100 \mathrm{mPa} \cdot \mathrm{s})$ of high-boiling PFPE's. The competition during droplet formation between viscous forces and interfacial tension can be expressed by the capillary number, $C a=U \mu / \gamma$, where $U$ is the linear flow velocity, $\mu$ is the viscosity of the carrier fluid, and $\gamma$ is the interfacial tension between the two fluids. At high $\mu$ and low $\gamma$, relatively low flow rates result in high $C a(>0.1)$, which can promote the laminar flow of parallel, immiscible streams. ${ }^{9,}{ }^{30}$ Droplet formation is discouraged at high $\mathrm{Ca}$ because the capillary velocity $(\gamma / \mu)$ is not fast enough relative to $U$ to relax the strained interface into droplets. ${ }^{30}$ Droplet formation is also hindered when the viscosity ratio, $\lambda=\mu_{\text {drop }} / \mu_{\text {carrier }}$, is very low $(<0.05) .{ }^{31}$ To rupture the interface at low viscosity ratios, the shear rate can be increased by 
narrowing microchannel dimensions $(<50 \mu \mathrm{m})$, but this can generate high pressures when viscous PFPE's are used as carrier fluids. Thus, to synthesize nanocrystals in droplets at high temperatures, a method to reproducibly generate droplets at high capillary numbers and low viscosity ratios must be developed.

In this work, we demonstrate successful droplet formation and flow in a high-temperature microreactor using solvents and conditions that are appropriate for the nanoliter-scale synthesis of CdSe nanocrystals. Using a stepped microstructure, controlled streams of octadecene droplets are reproducibly generated in perfluorinated polyether at low viscosity ratio and high $\mathrm{Ca}$. CdSe nanocrystals are synthesized at high temperature in droplet-based microreactors to demonstrate the compatibility of our droplet fluids. The benefits of performing high-temperature nanocrystal synthesis in self-contained nanoliter-scale reaction volumes are discussed in the context of other chemical and biochemical reactions where the physical, temporal, and thermal control and isolation of nanoliter-scale reaction volumes are critical elements. 
a

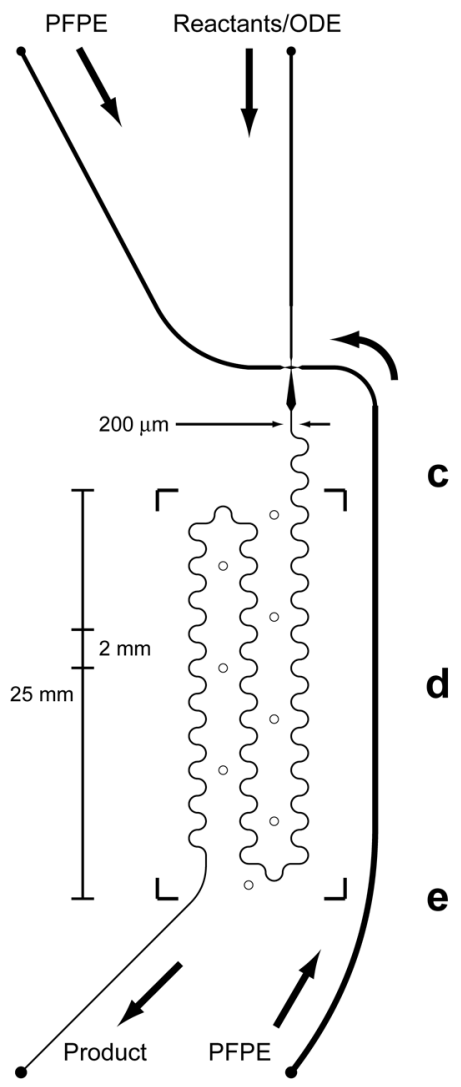

b

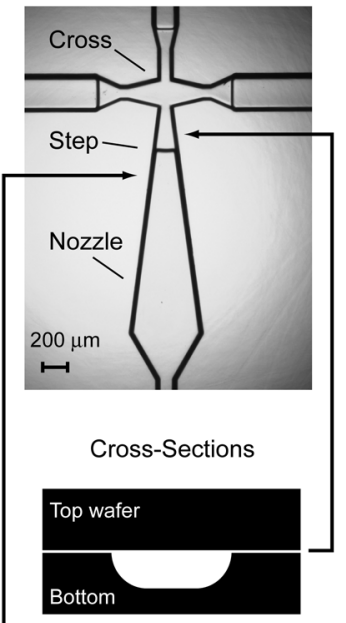

d

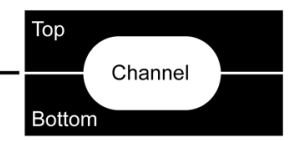

Side View

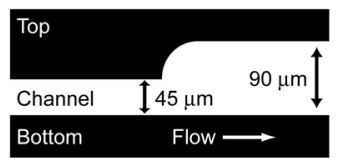

Fig. 1. Microreactor channel design with droplet jet injector. (a) Channel schematic showing dimensions, inlets $(\bullet)$, thermocouple wells $(\mathrm{O})$, and boundaries of Kapton heater (square brackets). (b) Optical micrograph of droplet injection cross. Octadecene is injected in the top channel, while the PFPE is injected in the side channels. The narrowest point is $160 \mu \mathrm{m}$ wide. (c) Lateral " $D$ "-shaped cross section of channel etched on the bottom wafer only. (d) Cross-section of ellipsoidal channel etched on both top and bottom wafers. (e) Axial cross-section showing the $45 \mu \mathrm{m}$ step up in channel height. 


\section{Experimental}

Microreactor design and fabrication. To make droplets of ODE in PFPE at high capillary numbers, we fabricated glass microreactors using the mask pattern shown in Fig. 1a. Droplets of the dispersed reactant phase are produced in the cross-shaped nanojet droplet generator (magnified in Fig. 1b), which is an extension of the designs developed by Tan et al. ${ }^{26,32}$ The perfluorinated polyether (PFPE) carrier fluid is injected into the side arms of the cross, while the dispersed reactant phase is injected at the top of the cross. The hydrodynamic focusing and shearing of the dispersed phase stream at the narrow, 160 $\mu \mathrm{m}$-wide constriction, coupled with the equilibrating effects of surface tension as the jet nozzle expands, leads to droplet production. From the end of the $2.4 \mathrm{~mm}$-long nozzle, the generated droplets then travel through a $200 \mu \mathrm{m}$-wide heated serpentine channel whose semi-circular turns are intended to induce mixing in the droplets. ${ }^{33}$ The angled brackets in Fig. 1a represent the boundaries of a 2.5 x $1 \mathrm{~cm}$ Kapton thin film heater (Minco) that heats the $107 \mathrm{~mm}$-long, $1.7 \mu \mathrm{L}$ reaction channel from the bottom of the chip.

To prevent continuous filaments of the dispersed phase from flowing laminarly through the entire channel at high $\mathrm{Ca}$, a sharp increase in height is fabricated $400 \mu \mathrm{m}$ after the beginning of the nozzle. Such an out-of-plane expansion has been shown to produce monodisperse emulsions in viscous carrier fluids, ${ }^{7}$ analogous to the function of the in-pane expansion. The locations of these "steps" in channel height are clearly visible as black lines in the micrograph in Fig. 1b. The cross-section illustrations in Fig. 1c-e show the geometrical details of the step in the nozzle.

Microfabrication. To fabricate this multi-level structure, two masks were used to pattern two separate wafers following a protocol published previously. ${ }^{34}$ The top mask is nearly identical to the continuous bottom mask (Fig. 1a) except that the top pattern does not extend to the cross region. Concentrated (49\%) HF was used to etch $1.1 \mathrm{~mm}$-thick borofloat glass wafers (Precision Glass \& Optics) to a depth of $45 \mu \mathrm{m}$ using standard planar photolithographic methods. The etched surfaces of the top and bottom 
wafers were placed in contact, and the channels were aligned manually under a microscope to a precision of $\pm 5 \mu \mathrm{m}$. The aligned wafers were thermally bonded to enclose the channels.

As seen in the axial cross-section (Fig. 1e), this procedure produces a step increase in channel height from 45 to $90 \mu \mathrm{m}$ after the cross. The isotropic wet etching results in channels with D-shaped cross sections in the single-etched cross region (Fig. 1c) and channels with ellipsoidal cross sections where two D-shaped channels are aligned (Fig. 1d). Channel widths refer to the maximum width.

Silanization. Stable droplet flow can only be obtained when the continuous phase (PFPE) preferentially wets the channel surface. ${ }^{35}$ Therefore, the glass surface of the microchannels was silanized with 1H,1H,2H,2H-perfluorodecyltrichlorosilane (FDTS, Lancaster), based on a procedure published by Srinivasan et al. ${ }^{36}$ The stability and effectiveness of this silane coating at high temperatures was especially sensitive to deviations from the following silanization procedure. After bonding, microchannels were cleaned and oxidized with piranha $\left(3: 1 \mathrm{vol} / \mathrm{vol}_{2} \mathrm{SO}_{4} / \mathrm{H}_{2} \mathrm{O}_{2}\right)$ at $80{ }^{\circ} \mathrm{C}$, then rinsed sequentially with Millipore water, isopropanol, and isooctane, and dried with a stream of filtered nitrogen. The silanization was performed under argon atmosphere, because FDTS reacts readily with water to form insoluble sol-gel aggregates. In a glovebox, $1.5 \mathrm{~mL}$ of a $0.66 \mathrm{mM}$ FDTS/isooctane solution was pumped through the channels over the course of 1 hour. The channels were then rinsed with isooctane, isopropanol, and water, then purged with nitrogen. Finally, the surface of the channels was dehydrated in a $120{ }^{\circ} \mathrm{C}$ oven for $12 \mathrm{~h}$ in order to drive cross-linking between adjacent siloxyl groups. Flat borofloat substrates silanized using this procedure had static water/air contact angles of $115^{\circ}$ as measured by a Kruss Contact Angle Measuring System. Silanization of microreactor walls was verified by observing the preferential wetting of the fluorinated phase under a microscope.

Droplet production \& characterization. Droplets were generated by pumping filtered ODE and Fomblin Y 06/6 PFPE through a freshly silanized chip at rates from 0.1 to $20 \mu \mathrm{L} / \mathrm{min}$ using three, 500$\mu 1$ Hamilton gastight syringes loaded in two syringe pumps (BAS). Syringes were interfaced to the chip 
via PEEK fittings (Upchurch) and a custom aluminum manifold.

Capillary numbers were calculated using the average linear fluid velocity at the nanojet constriction and the values of $\mu$ and $\gamma$ at $20{ }^{\circ} \mathrm{C}$. The viscosity of Fomblin Y 06/6 (avg. MW = 1800) at $20{ }^{\circ} \mathrm{C}$ is $113 \mathrm{mPa} \cdot \mathrm{s}$, compared to $4 \mathrm{mPa} \cdot \mathrm{s}$ for octadecene, resulting in $\lambda=0.035 .{ }^{37}$ The interfacial energy between ODE and Fomblin Y $06 / 6$ at $20{ }^{\circ} \mathrm{C}$ is $8.3 \pm 0.3 \mathrm{mN} / \mathrm{m}$, as measured with a KSV Sigma 701 tensiometer using the Du Nouy ring method. ${ }^{38}$

At high $\mathrm{Ca}$, laminar flow was observed and, once established, remained stable even after conditions were returned to those known to favor droplet formation. To prevent such hysteresis, the ODE flow was stopped each time the flow rate was changed until the nanojet was purged of ODE. After restarting the ODE flow, the system was allowed to equilibrate for 2 min before observations and images were recorded.

Droplet formation was observed at room temperature using a Nikon Eclipse E800 microscope. Images were captured with an Evolution MP CCD camera (MediaCybernetics) with an integration time of $1.6 \mathrm{~ms}$. Image Pro Plus software (MediaCybernetics) was used to acquire droplet images and perform droplet sizing. Droplet formation at high temperature was characterized through the objectives and with low-resolution images.

\section{Nanocrystal Synthesis.}

Reagents. Cadmium oxide powder $(99.99+\%)$, selenium powder $(99.999 \%)$, oleic acid $(90 \%)$, anhydrous isooctane (2,2,4-trimethylpentane, 99.8\%), HPLC-grade acetone (99.9+\%), 1-octadecene (>95\%), and Fomblin Y 06/6 perfluorinated polyether were purchased from Sigma-Aldrich. Tri-noctylphosphine $(97+\%)$ was purchased from Strem. Fomblin Y06/6 and all reaction solutions were degassed under vacuum to remove air and water and then stored in an argon-filled glovebox. Before

synthesis, all solutions were degassed again to prevent bubble evolution and filtered with $0.45 \mu \mathrm{m}$ 
syringe filters (Pall) to prevent clogging.

Stock solution preparation. $60 \mathrm{mg}$ of $\mathrm{CdO}, 1.0 \mathrm{~g}$ of oleic acid, and $1.90 \mathrm{~g}$ of octadecene (ODE) were heated to $200{ }^{\circ} \mathrm{C}$ under argon to form a clear yellow cadmium oleate stock solution that contained 132 $\mathrm{mM}$ cadmium with 7:1 oleic acid:Cd mole ratio. A $43 \mathrm{mM}$ selenium stock solution was prepared by mixing $0.65 \mathrm{~g}$ of degassed octadecene with $0.034 \mathrm{~g}$ of a solution of tri- $n$-octylphosphine selenide (TOPSe) in tri- $n$-octylphosphine (TOP) that was $10 \%$ Se by weight. For some experiments, the $\mathrm{Cd}$ and Se stock solutions were diluted three-fold by adding additional octadecene.

Synthesis apparatus \& procedure. In a typical experiment, Harvard Apparatus Pump 11 Pico Plus syringe pumps injected separate streams of $\mathrm{Cd}$ and Se stock solution at equal rates into a PEEK tee. The combined $66 \mathrm{mM} \mathrm{Cd}, 22 \mathrm{mM}$ Se solution, referred to here as the "ODE flow," was pumped into the reactant inlet of the chip. Fomblin Y 06/6 PFPE was pumped into the chip using a Harvard PHD2000 pump, with the ODE:PFPE flow rate ratio kept constant at 1:4 for all nanocrystal syntheses. Pumping and all other chip functions were computer-controlled through a master LabView virtual instrument (VI) (National Instruments).

The thin-film heater was powered by a programmable power supply (Instek) whose voltage was PID-controlled by the master LabView VI. Reactor set point temperatures ranged from 240 to $300^{\circ} \mathrm{C}$ and were measured by 36 gauge thermocouples (Omega) embedded at channel depth in drilled holes in the center of the heated region. Thermocouples placed near the edges of the heater measured a drop of approximately $50^{\circ} \mathrm{C}$ from the center.

The droplet residence time at $1.25 \mu \mathrm{L} /$ min total flow rate was measured by timing many individual droplets as they traveled through the reaction channel. Residence times for high flow rates were extrapolated from this value, and measurements at medium flow rates validated the accuracy of this method. Droplets of product solution were output into a capillary flow cell attached to a CCD spectrometer (Ocean Optics) for fluorescence detection (380 nm excitation). Reaction product was collected from the end of the capillary in a vial under nitrogen. 
Cleaning \& characterization. In a glovebox, the product mixture was centrifuged, and the top, colored ODE phase was separated, precipitated with acetone, and centrifuged again to form a clean pellet of nanocrystals. A minimum of $20 \mu \mathrm{l}$ of nanocrystal solution (100 $\mu$ l of raw product mixture) was necessary for adequate pellet formation. Cleaned nanocrystals were characterized with a Tecnai $\mathrm{G}^{2} 20$ transmission electron microscope (FEI) at $200 \mathrm{KV}$ beam acceleration. UV-visible absorption spectra on cleaned aliquots were recorded in quartz cuvettes on an Agilent 8453 spectrophotometer. 


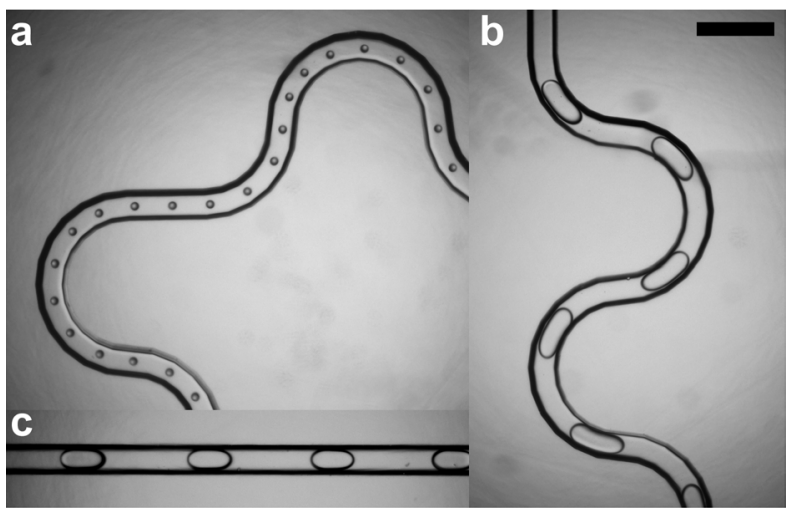

Fig. 2. Droplet images in main channel at the following ODE:PFPE flow rates: (a) $0.1: 2 \mu \mathrm{L} / \mathrm{min}$, (b) \& (c) $1: 2 \mu \mathrm{L} / \mathrm{min}$. Droplets were generated at capillary numbers of (a) 0.075 , and (b) \& (c) 0.11 . Scale bar $=500 \mu \mathrm{m}$.

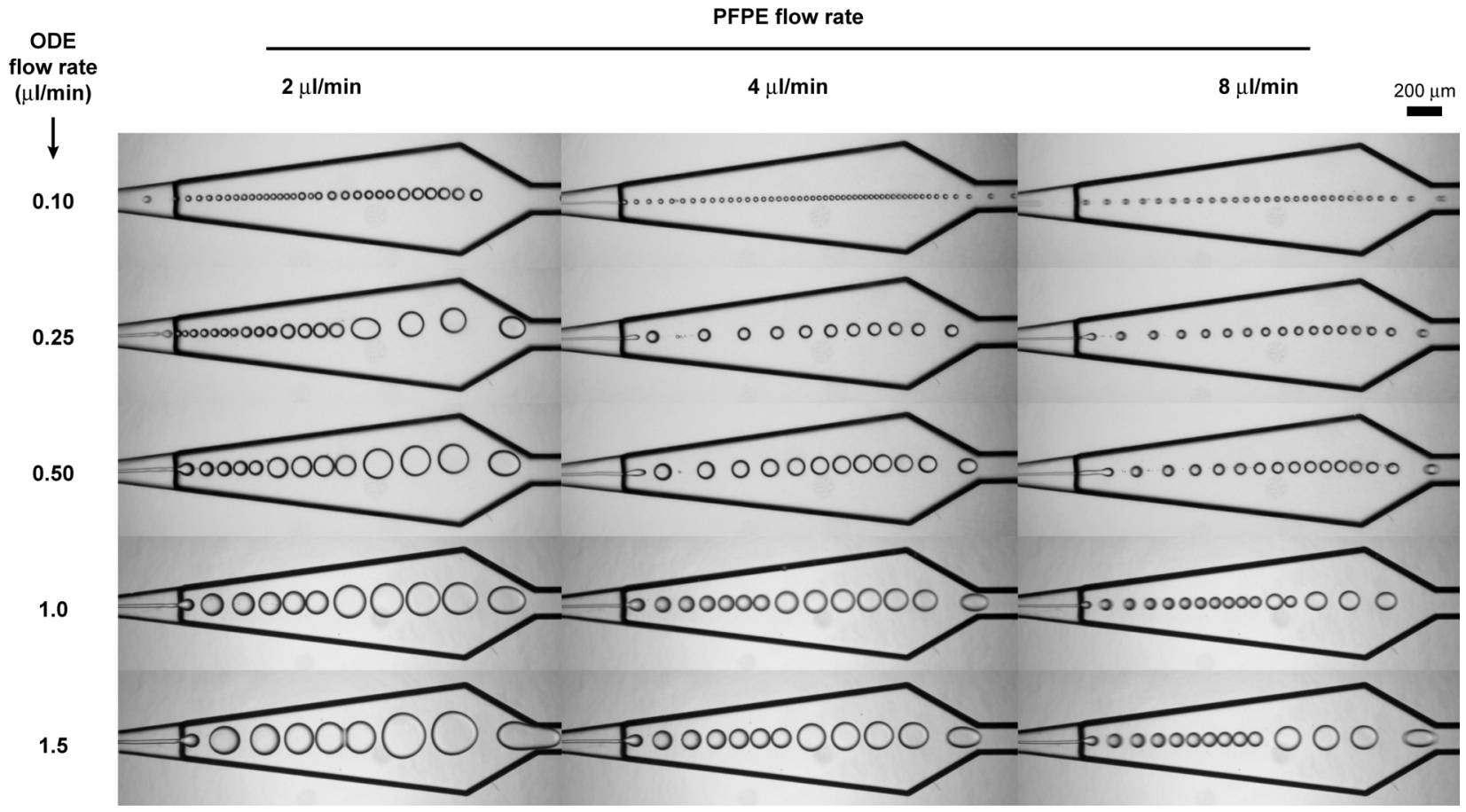

Fig. 3. Optical micrographs of droplet formation at the indicated ODE and PFPE flow rates. The maximum Ca shown is 0.34 for 1.5:8 ODE:PFPE. 


\section{Results and Discussion}

Droplet formation at ambient temperature. Using a nanojet injector modified with a downstream step

(Fig. 1), our microreactor successfully generated steady, controlled streams of ODE droplets in PFPE carrier fluid. Fig. 2 presents optical micrographs of small, spherical droplets and large, oblong droplets flowing through the main reactor channel without interacting with each other. The $53 \mu \mathrm{m}$-diameter, 78 pL droplets in Fig. $2 \mathrm{a}$ were formed with 0.1:2 $\mu \mathrm{L} / \mathrm{min}$ ODE:PFPE and the $300 \mu \mathrm{m}$-long, $135 \mu \mathrm{m}$-wide droplets in Fig. 2c were formed with 1:2 $\mu \mathrm{L} / \mathrm{min}$ ODE:PFPE.

The expansion step in particular allowed the nanojet to generate droplets over a wide range of flow rates and capillary numbers $(\mathrm{Ca})$. Fig. 3 shows optical micrographs of droplets being generated in the nanojet nozzle at room temperature with ODE flow rates ranging from 0.1 to $1.5 \mu \mathrm{L} / \mathrm{min}$ and total PFPE flow rates from 2 to $8 \mu \mathrm{l} / \mathrm{min}$. In these frames, the capillary number, which is proportional to total flow rate, ranges from $C a=0.075$ to 0.34 . As illustrated in Fig. 3, droplet size increases with increasing ODE flow rates and decreasing PFPE flow rates. In Fig. 3, droplet diameters range from 37 $\mu \mathrm{m}(0.1: 8 \mu \mathrm{L} / \mathrm{min}$ ODE:PFPE) to $284 \mu \mathrm{m}$ (1.5:2 ODE:PFPE), corresponding to volumes of $27 \mathrm{pL}$ to 5 $\mathrm{nL}$, respectively. ${ }^{39}$ When the total flow rate was kept constant, droplet size increased with the "ODE fraction" -- the ratio of the ODE volumetric flow rate to the total flow rate. When the ODE fraction was kept constant, the final droplet size exiting the nozzle did not vary significantly with the total flow rate. Many frames in Fig. 3 also show the consistent, sequential fusion of smaller droplets into larger droplets, ${ }^{39}$ which enables the predictable formation of larger droplets without increasing the ODE fraction. Thus, by using this modified nanojet, we can tune the size of ODE droplets independently from flow rate, even at high capillary numbers.

As the ODE fraction and flow rate are increased, droplet generation passes through four distinct phases: (I) droplet separation before the step, (II) step-induced separation in the first half of the nozzle, 
(III) separation in the second half of the nozzle, and (IV) laminar flow. At the lowest ODE and PFPE flow rates (Phase I, e.g. 0.1:2 ODE:PFPE in Fig. 3), droplets formed before the microfabricated step in the nanojet nozzle. At moderate flow rates and ODE fractions (Phase II, e.g. 1:2 ODE:PFPE), the ODE stream formed a thin filament that only broke off into droplets at the step. Occasionally, the filament extended slightly past the step, as shown with 0.5:8 ODE:PFPE. In these cases, the microfabricated step instigated long-wavelength undulations that eventually degenerated into droplets due to Rayleigh capillary instability. ${ }^{40}$ At higher ODE fraction and flow rates (Phase III), laminar filaments broke off into droplets in the second half of the nozzle or at the head of the reaction channel. At the highest ODE fraction and total flow rates (Phase IV), filaments extended across the entire nozzle and through the entire length of the reaction channel.

The phase diagrams in Fig. 4 summarize these four regimes as a function of ODE fraction and $\mathrm{Ca}$. Here we consider Phases I and II to be clean droplet separation and Phase III to be the transition region before the laminar flow of Phase IV. Fig. 4a clearly shows the regions of clean droplet separation at low ODE fraction or low $C a$, as well as the regions of laminar flow at high ODE fraction and $\mathrm{Ca}$. In comparison, using a $45 \mu \mathrm{m}$-deep microreactor without a step (Fig. 4b), the same sequence of phases is observed, but the area of the phase diagram that cleanly generates droplets shrinks to a small fraction of the analogous area in Fig. 4a. With the step, reproducible droplet separation was observed at $C a$ up to 0.81 at ODE fraction $=0.11$. At the same ODE fraction without the step, clean separation was only observed up to $C a=0.08$, and did not extend past $C a=0.22$ even at lower ODE fractions. With the step, droplet formation was observed at ODE fractions up to 0.6 at $C a=0.13$, compared to ODE fraction $=0.167$ without the step. These dramatic differences explain why laminar flow was observed for most $\mathrm{Ca}$ when flowing ODE and PFPE in nanojet structures without the step modification. As intended, the step expands the available phase space for droplet formation, particularly along the $\mathrm{Ca}$ axis. 


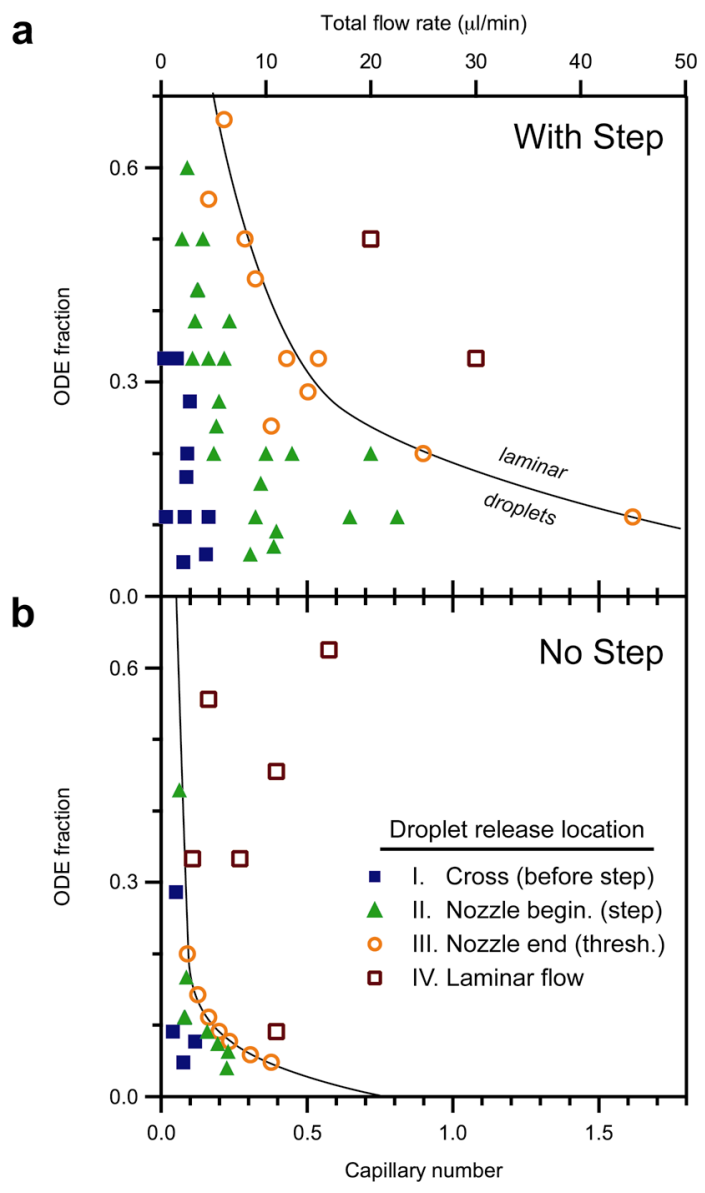

Fig. 4. Phase diagrams showing the location of droplet separation for (a) a $90 \mu \mathrm{m}$-deep channel with a $45 \mu \mathrm{m}$ step, and (b) an equivalent $45 \mu \mathrm{m}$ deep channel with no step. The line drawn through the threshold region (III) distinguishes the laminar flow regime (IV) from droplet regime (I \& II).

Our step-modified nanojet generated droplets at $C a(0.81)$, comparable to the highest values reported for other microfabricated devices, and at a significantly lower viscosity ratio. Zheng et al. observed droplet formation in viscous carrier fluids $(\lambda=0.11)$ up to $C a=0.11,{ }^{11}$ which is consistent with the maximum $C a=0.08$ that we observed at the same volume fraction without the step. Anna et al. demonstrated droplet formation up to $C a \sim 1.0,{ }^{9}$ but with a much less viscous carrier fluid ( $\left.\mu=6 \mathrm{mPa} \cdot \mathrm{s}\right)$ and a viscosity ratio $(\lambda=0.17)$ five times higher than the $\lambda$ for ODE/PFPE.

Clearly, the microfabricated step in our nanojet device plays an integral role in disrupting the laminar filaments observed at high $\mathrm{Ca}$. The rapid expansion in channel height, ${ }^{7}$ coupled with the sudden reduction in flow velocity and $\mathrm{Ca},{ }^{41}$ forces the end of the filament to expand into a bulbous 
shape at the step. As observed by Stone et al., the inhomogeneous Laplace pressure resulting from this bulbous end, along with the sharp corner of the step, generates a narrow "neck" that is a prerequisite for droplet formation. ${ }^{41}$ The decreased flow velocity relative to the capillary relaxation velocity allows surface tension time to pinch off the neck and release droplets.

High temperature droplet formation. Nanoliter droplets of ODE were also generated successfully in PFPE when the reaction channel was heated to temperatures up to $300{ }^{\circ} \mathrm{C}$. Because surface tension and viscosity are temperature-dependent, the nanojet injector itself was not directly heated (Fig. 1a) in order to maintain the droplet generation behavior previously observed at room temperature. As the droplets entered the heated reaction channel, thermocapillary effects ${ }^{42}$ resulting from the temperature gradient caused small droplets to fuse. The resulting droplets filled the width of the channel, which had the beneficial effect of making the droplets flow closer to the average linear velocity in the channel. The aspect ratios of the resulting droplets were 1-2 under most conditions, but increased to 4 at extremely low flow rates $(\sim 1 \mu \mathrm{L} / \mathrm{min})$. Outside of the regions of steeply increasing temperature, droplets did not combine again in the reaction channel. The perfluoroalkysilane coatings on the channel walls maintained their surface properties for approximately 5 hours, after which the ODE droplets began wetting the surface of the channel, resulting in irregular or laminar flow. Contact angle experiments on flat substrates confirmed that after heating for $>5$ hours, the perfluoroalkylsilanes were almost completely removed in the presence of PFPE at high temperature. 
High temperature nanocrystal synthesis. After confirming that droplet flow remained stable at high temperatures and capillary numbers, we demonstrated the ability to synthesize CdSe nanocrystals at high temperature in nanoliter-scale droplets. $\mathrm{Cd} / \mathrm{Se}$ precursor solutions formed stable, distinct, nanoliter droplets in PFPE, and the solutions reacted consistently when heated to produce faint orange or red droplets of nanocrystal solution. The PFPE did not dissolve or mix with any of the reagents in the high temperature microreactor and did not show the typical signs of thermal degradation, ${ }^{43}$ such as vigorous bubbling. ${ }^{44}$ The nanocrystal/ODE phase was easily separated from the clear PFPE phase for cleaning. The TEM micrographs of cleaned product in Fig. 5 show nicely ordered arrays of 3.8 nm-diameter CdSe nanocrystals synthesized in ODE droplets for $10 \mathrm{~s}$ at $300^{\circ} \mathrm{C}$. A high-resolution image (Fig. 5 inset) of a $3.4 \mathrm{~nm}$-diameter nanoparticle grown at $260{ }^{\circ} \mathrm{C}$ displays the characteristic hexagonal symmetry of the wurtzite crystal phase typically found in CdSe nanocrystals synthesized at high temperature.

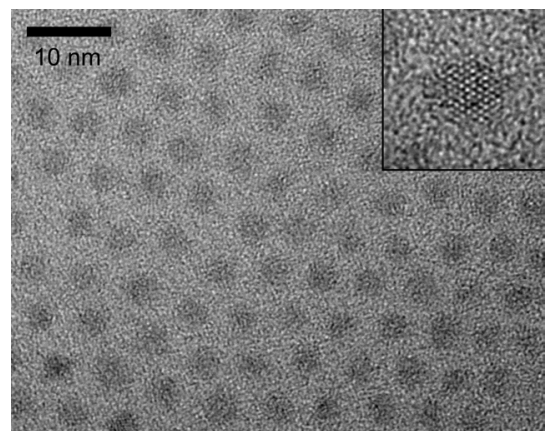

Fig. 5. Transmission electron micrographs of CdSe nanocrystals synthesized in droplets of ODE in PFPE in a $290^{\circ} \mathrm{C}$ microreactor. Inset: high resolution image of a $3.4 \mathrm{~nm}$-diameter nanocrystal.
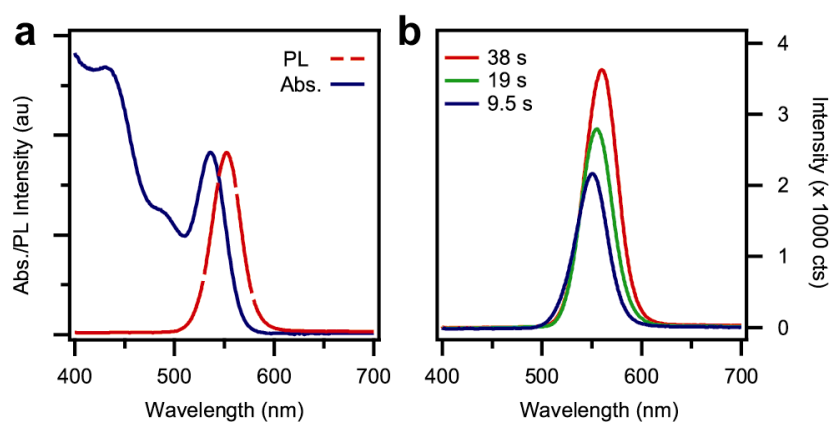

Fig. 6. (a) On-line photoluminescence spectrum and off-line absorption spectrum of nanocrystals grown in droplets for $19 \mathrm{~s}$ at $280^{\circ} \mathrm{C}$. (b) On-line photoluminescence spectra of nanocrystals grown at $290^{\circ} \mathrm{C}$ with three different flow rates and residence times.

Fig. 6a shows absorption and photoluminescence (PL) spectra for nanocrystals synthesized in nanoliter-scale droplets for $19 \mathrm{~s}$ at $280{ }^{\circ} \mathrm{C}$. To synthesize these nanocrystals, $132 \mathrm{mM} \mathrm{Cd}$ and $43 \mathrm{mM} \mathrm{Se}$ stock solutions were injected at $0.25 \mu \mathrm{L} / \mathrm{min}$ each, while the PFPE was pumped at $2 \mu \mathrm{L} / \mathrm{min}$. The offline absorption and on-line fluorescence spectra exhibit the sharp, distinctive peaks of fairly monodisperse nanocrystals. The PL full width at half maximum (fwhm) of $34 \mathrm{~nm}$ is comparable to those seen with the analogous flask and continuous flow syntheses and demonstrates the superior optical 
properties of nanocrystals synthesized in high temperature microreactors, compared to those synthesized in low temperature chips.

Fig. 6b shows on-line PL spectra for nanocrystals synthesized at $290{ }^{\circ} \mathrm{C}$ at three different residence times, which varied with total flow rate. The fluorescence spectra in Fig. 6b show that, as the residence time increases, the peak intensity increases, and the emission peaks shift from 550 to 555 to $560 \mathrm{~nm}$ as the residence times are increased from 9.5 to 19 to $38 \mathrm{~s}$, respectively. The relatively small shift in the emission peaks implies that the reaction is close to completion after only $9.5 \mathrm{~s}$ of growth. This agrees with our general observation that $\mathrm{CdSe}$ reactions involving Cd-oleate occur very rapidly due to the weak coordination of the oleic acid surfactant. In addition, the relatively high oleic acid:Cd ratios (7:1) used are believed to encourage Ostwald ripening, ${ }^{28}$ which can explain why the PL peaks in this particular reaction system are not significantly narrower when using droplets.

The synthesis of CdSe nanocrystals in discrete, uniformly flowing, nanoliter-volume droplets theoretically allows for the precise characterization of such rapid growth on the single-drop level. The PL spectra in Fig. 6b were recorded on-line as droplets of nanocrystal product flowed out of the microreactor. Spectra of droplets in the capillary flow cell were resolved at time resolutions down to $250 \mathrm{~ms}$. Fig. 7 shows the peak wavelength and intensity time traces for droplets of nanocrystals flowing at $0.5: 2 \mu \mathrm{L} / \mathrm{min}$ ODE:PFPE at $290{ }^{\circ} \mathrm{C}$, which corresponds to the $19 \mathrm{~s}$ spectrum in Fig. 6b. The intermittent spikes in the peak wavelength and intensity traces show individual droplets as they passed through the detector. Over the course of the $120 \mathrm{~s}$ window shown in Fig. 7, the PL intensity of each droplet was very reproducible, and the peak wavelengths did not shift significantly -- the $\pm 0.3 \mathrm{~nm}$ variation was comparable to the resolution of the spectrometer and precision of peak-fitting algorithm. The length of the droplets $(\sim 1 \mathrm{~s})$ and the occasional variations in their periodicity are consistent with the observation that droplets from the microreactor combined on the order of 10 times outside of the reactor as they flowed through the $500 \mu \mathrm{m}$-wide exit via and the external capillary. Because this abrupt widening occurred at room temperature, this droplet fusion would not affect growth kinetics or broaden 
the PL peaks of the resulting drops under stable growth conditions. PL peaks for droplets of nanocrystals were observed to remain stable over several hours and hundreds of droplets. On-line spectroscopy is an invaluable diagnostic tool for microreactor synthesis, because the growth and PL spectra of nanocrystals are extremely sensitive to changes in channel conditions caused by bubble formation, channel obstruction, degrading surface properties, and most significantly, deposition of nanocrystals on channel walls. The stable traces confirm our visual observations that, over the span of four hours, none of these phenomena occurred in amounts significant enough to perturb high temperature droplet synthesis. In contrast, using analogous conditions in a continuous flow reactor, an opaque layer of nanocrystals forms after $\sim 20$ minutes at the head of the heated region, resulting in the shifting and broadening of the PL peak.

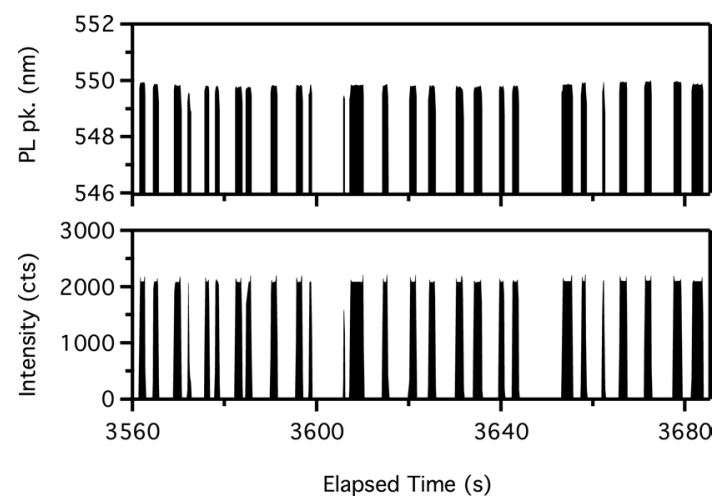

Fig. 7. Photoluminescence time traces showing peak wavelength (top) and intensity (bottom) data as individual drops of synthesized nanocrystals pass through the flow cell. The flow rates were $0.25: 0.25: 2$ $\mu \mathrm{L} / \mathrm{min}$ Cd:Se:PFPE. Spectral data and conditions correspond to the $19 \mathrm{~s}$ run from Fig. 6 b.

Fig. 8 shows that, with the stability of our droplet microreactor, we can test four different flow rates and three different temperatures in a single 4-hour experiment. In order to obtain a wider range of nanocrystal sizes, the concentrations of the $\mathrm{Cd}$ and Se stock solutions were diluted three-fold compared to the synthesis associated with Fig. 6. Fig. 8 shows that, as the total flow rates are halved sequentially from 10 to $1.25 \mu \mathrm{L} / \mathrm{min}$ and the temperature is increased in $10{ }^{\circ} \mathrm{C}$ increments from 270 to $290{ }^{\circ} \mathrm{C}$, the nanocrystal PL peak wavelength increases in distinct steps that represent increases in nanocrystal diameter. The steps in these time traces are much sharper and the $\sim 2-3$ min equilibration time is 4 times 
shorter than the $\sim 10$ min previously reported ${ }^{13}$ with continuous flow reactors because there is significantly less dispersion when using droplets. The remaining equilibration and lag times are attributed to the time needed stabilize the pressure and droplet flow as well as the relatively large volume $(20 \mu \mathrm{L})$ of the capillary connecting the chip outlet and the flow cell. The fwhm traces (Fig. 8b) demonstrate that the size distribution increases as the residence time and nanocrystal size are increased. This behavior is consistent with the rapid Ostwald ripening we observe with oleic acid. The broadening could have been exacerbated by the fact that the least consistent droplet fusion occurred at the lowest flow rate $(1.5 \mu \mathrm{L} / \mathrm{min})$. The stable PL peak wavelength time traces at each condition, even after several hours of synthesis, are possible largely because the encapsulation of growing nanocrystals in droplets prevents them from interacting with the channel walls.

The spectral data recorded in the single run shown in Fig. 8a can be plotted as a peak wavelength vs. residence time graph (Fig. 8d) that shows kinetic data for nanocrystal growth at three different temperatures. The three temperatures display similar growth curves that flatten at increased residence time, but the higher temperatures shift the traces to higher wavelengths. These curves are similar to the kinetic data reported in previous high-temperature nanocrystal syntheses, ${ }^{28}$ which validates our experimental methods. More significantly, Fig. 8 demonstrates that such data can be collected rapidly and precisely in large part because our microfluidic droplet reactor offers the flexibility and stability to test a wide range of conditions. Capillary numbers reached up to $C a=0.36$ and temperatures reached up to $290{ }^{\circ} \mathrm{C}$ - conditions not readily accessible using other droplet technologies. The isolation of nanocrystals in discrete droplets allowed growth kinetics to be observed at short time scales and allowed the reactor to be run continuously for four hours at high temperature without cleaning. Thus, our ability to generate droplets of precursor solution in PFPE at high $\mathrm{Ca}$ significantly improves our ability to synthesize nanocrystals in high-temperature microreactors with a number of temperatures, residence times, and different reaction schemes. 
a

b

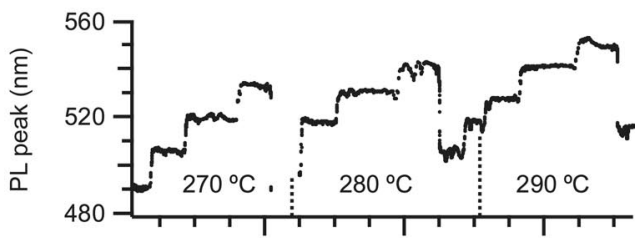

C
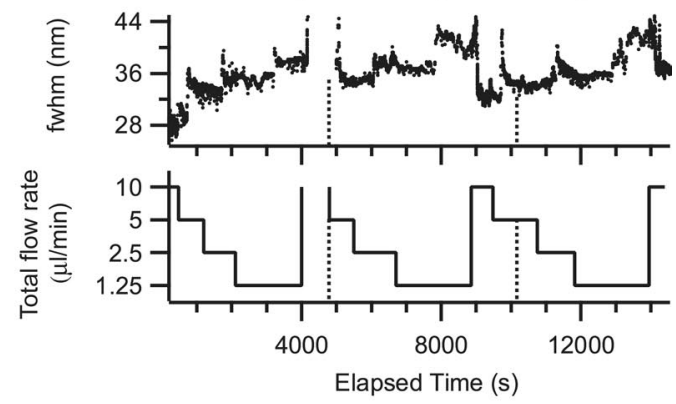

d

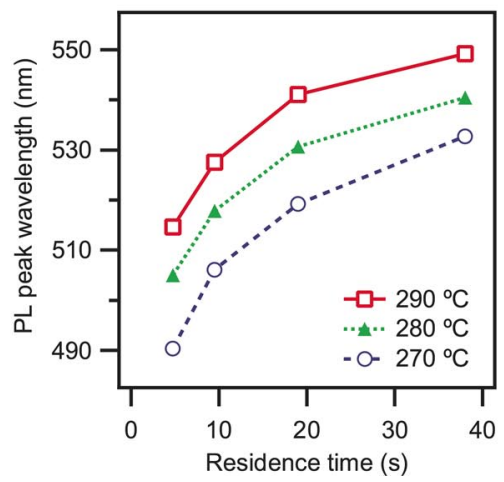

Fig. 8. Photoluminescence time traces of nanocrystal drops demonstrate how (a) peak wavelength and (b) full width at half max vary with (c) total flow rate at three different temperatures. The discontinuity at $4000 \mathrm{~s}$ marks a break for syringe refilling. (d) Kinetic growth curves (peak wavelength vs. residence time) can be obtained for three temperatures using the data from (a) and (c). 


\section{Conclusion}

We have demonstrated high-temperature synthesis of nanocrystals in a liquid-liquid segmented flow nanojet microreactor. Because the pyrolytic synthesis of high-quality nanocrystals requires extreme conditions, several key developments are introduced to integrate the CdSe nanocrystal synthesis with droplet-flow microreactors. First, organic and perfluorinated solvents are used to produce two-phase flows in microfluidic devices. Second, in order to flow droplets over a wide range of flow rates, a nanojet injector with an expansion step is used to produce droplets at a low viscosity ratio $(\lambda=$ $0.035)$ and at capillary numbers (0.81) comparable to the highest reported values. The ability to form droplets at high $\mathrm{Ca}$ with immiscible, high-boiling solvents allows our microfluidic devices to maintain reproducible droplet flow at high-temperature. All of these developments are essential for the synthesis of inorganic nanocrystals in nanoliter droplets of organic solvents in perfluorinated fluids.

While the nanocrystals used to demonstrate this novel droplet reactor are comparable in monodispersity to those made in flask or continuous flow reactors, future optimization of precursor and surfactant systems, as well as the integration of on-chip mixing, should allow for the synthesis of more monodisperse particles. On-chip optical spectroscopy ${ }^{17}$ will allow the low-latency analysis of single drops, which will facilitate high-throughput screening of nanocrystals and growth conditions. While gas-liquid and liquid-liquid segmented flow reactors are complementary techniques, the isolation of nanoliter droplets from channel walls makes droplet-based reactors more robust, more general, and more suitable for rapid reactions. The current durability of our droplet reactors determined by the $\sim 5$ hour lifetime of the fluoroalkylsilane coating, but stabilization of the unfluorinated $\mathrm{C}_{1}$ and $\mathrm{C}_{2}$ atoms on FDTS should allow for extended droplet experiments.

The successful use of PFPE as the carrier fluid for nanoliter droplets has implications for other chemical or biochemical syntheses. With the appropriate PFPE carrier fluid, many reactions in aqueous or organic solution can be encapsulated in nanoliter droplets and heated to the desired reaction temperatures in order to exploit the advantages of microfluidic reactors. These capabilities should be 
useful in studies of a wide variety of chemical and biochemical reactions where small reaction volumes and small numbers of reactant molecules, isolation from container walls and other reaction volumes, and fine control of temperature and other conditions are of critical importance.

Acknowledgements. Chip fabrication was performed at the UC-Berkeley Microfabrication Laboratory. This work was supported by the donors of the Center for Analytical Biotechnology and by the Director, Office of Energy Research, Office of Science, Division of Materials Sciences, of the U.S. Department of Energy under Contract No. DE-AC03-76SF00098. 


\section{References}

(1) Lopez-Quintela, M. A.; Tojo, C.; Blanco, M. C.; Rio, L. G.; Leis, J. R. Curr. Opin. Colloid Interface Sci. 2004, 9, 264-278.

(2) Jensen, K. F. Chem. Eng. Sci. 2001, 56, 293-303.

(3) Haswell, S. J.; Middleton, R. J.; O'Sullivan, B.; Skelton, V.; Watts, P.; Styring, P. Chem.

Commun. 2001, 391-398.

(4) Pennemann, H.; Watts, P.; Haswell, S. J.; Hessel, V.; Lowe, H. Org. Process Res. Dev. 2004, 8, $422-439$.

(5) Burns, J. R.; Ramshaw, C. Lab Chip 2001, 1, 10-15.

(6) Lagally, E. T.; Emrich, C. A.; Mathies, R. A. Lab on a Chip 2001, 1, 102-107.

(7) Sugiura, S.; Nakajima, M.; Iwamoto, S.; Seki, M. Langmuir 2001, 17, 5562-5566.

(8) Thorsen, T.; Roberts, R. W.; Arnold, F. H.; Quake, S. R. Phys. Rev. Lett. 2001, 86, 4163-4166.

(9) Anna, S. L.; Bontoux, N.; Stone, H. A. Appl. Phys. Lett. 2003, 82, 364-366.

(10) Grodrian, A.; Metze, J.; Henkel, T.; Martin, K.; Roth, M.; Kohler, J. M. Biosens. Bioelectron. 2004, 19, 1421-1428.

(11) Zheng, B.; Tice, J. D.; Ismagilov, R. F. Anal. Chem. 2004, 76, 4977-4982.

(12) Shestopalov, I.; Tice, J. D.; Ismagilov, R. F. Lab Chip 2004, 4, 316-321.

(13) Chan, E. M.; Mathies, R. A.; Alivisatos, A. P. Nano Lett. 2003, 3, 199-201.

(14) DeMello, J.; DeMello, A. Lab Chip 2004, 4, 11N-15N.

(15) Yen, B. K. H.; Stott, N. E.; Jensen, K. F.; Bawendi, M. G. Adv. Mater. 2003, 15, 1858-1862.

(16) Nakamura, H.; Yamaguchi, Y.; Miyazaki, M.; Maeda, H.; Uehara, M.; Mulvaney, P. Chem. Commun. 2002, 2844-2845.

(17) Krishnadasan, S.; Tovilla, J.; Vilar, R.; deMello, A. J.; deMello, J. C. J. Mater. Chem. 2004, 14, 
2655-2660.

(18) Wang, H. Z.; Li, X. Y.; Uehara, M.; Yamaguchi, Y.; Nakamura, H.; Miyazaki, M. P.; Shimizu, H.; Maeda, H. Chem. Commun. 2004, 48-49.

(19) Okuyama, K.; Lenggoro, I. W. Chem. Eng. Sci. 2003, 58, 537-547.

(20) Levenspiel, O., The Chemical Reactor Omnibook. OSU Book Stores: Corvallis, OR, 1979; pp. 64.1-64.20.

(21) Yen, B. K. H.; Gunther, A.; Thalmann, M.; Bawendi, M. G.; Jensen, K. F. 8th International Conference on MicroTAS, Malmo, Sweden, 2004. Vol. 2, pp 126-129.

(22) Gunther, A.; Khan, S. A.; Thalmann, M.; Trachsel, F.; Jensen, K. F. Lab Chip 2004, 4, 278-286.

(23) Khan, S. A.; Gunther, A.; Schmidt, M. A.; Jensen, K. F. Langmuir 2004, 20, 8604-8611.

(24) Harries, N.; Burns, J. R.; Barrow, D. A.; Ramshaw, C. Int. J. Heat Mass Transfer 2003, 46, 3313-3322.

(25) Tice, J. D.; Song, H.; Lyon, A. D.; Ismagilov, R. F. Langmuir 2003, 19, 9127-9133.

(26) Tan, Y. C.; Collins, J.; Lee, A. P. 7th International Conference on MicroTAS, Squaw Valley, CA, 2003. Vol. 2, pp 963-966.

(27) Hung, L. H.; Tseng, W. Y.; Choi, K.; Tan, Y. C.; Shea, K. J.; Lee, A. P. 8th International Conference on MicroTAS, Malmo, Sweden, 2004. Vol. 1, pp 539-541.

(28) Bullen, C. R.; Mulvaney, P. Nano Lett. 2004, 4, 2303-2307.

(29) Yu, W. W.; Peng, X. Angew. Chem. Int. Ed. 2002, 41, 2368-2371.

(30) Tice, J. D.; Lyon, A. D.; Ismagilov, R. F. Anal. Chim. Acta 2004, 507, 73-77.

(31) Bentley, B. J.; Leal, L. G. J. Fluid Mech. 1986, 167, 241-283.

(32) Tan, Y. C.; Fisher, J. S.; Lee, A. I.; Cristini, V.; Lee, A. P. Lab Chip 2004, 4, 292-298.

(33) Song, H.; Tice, J. D.; Ismagilov, R. F. Angew. Chem. Int. Ed. 2003, 42, 768-772.

(34) Simpson, P. C.; Roach, D.; Woolley, A. T.; Thorsen, T.; Johnston, R.; Sensabaugh, G. F.; Mathies, R. A. Proc. Natl. Acad. Sci. U. S. A. 1998, 95, 2256-2261. 
(36) Srinivasan, U.; Houston, M. R.; Howe, R. T.; Maboudian, R. J. Microelectromech. Syst. 1998, 7, 252-260.

(37) Specified by manufacturer.

(38) Harkins, W. D.; Jordan, H. F. J. Am. Chem. Soc. 1930, 52, 1751-1772.

(39) The volume of the larger droplets was calculated assuming that they were cylindrical disks of channel height. The smaller droplets were observed to be spherical. Area measurements of combining drops confirmed these droplet shapes, as well as the fact that droplets combine most often in pairs and, under certain conditions, in groups of three.

(40) Rayleigh, L. Proc. R. Soc. London 1879, 29, 71-97.

(41) Stone, H. A.; Bentley, B. J.; Leal, L. G. J. Fluid Mech. 1986, 173, 131-158.

(42) Sammarco, T. S.; Burns, M. A. AIChE J. 1999, 45, 350-366.

(43) While the metal oxides of hard Lewis acids $\left(\mathrm{Al}^{3+}, \mathrm{Fe}^{3+}\right)$ have been shown to heterogeneously catalyze the thermal degradation of certain PFPE's after an hour-long induction period, Fomblin $\mathrm{Y}$ is stable under such conditions. Furthermore, it is unlikely that the Cd-oleate complexes or nanocrystals in solution would catalyze the PFPE degradation due to the (1) soft Lewis acidity of $\mathrm{Cd}^{2+},(2)$ the low Cd surface concentration at the ODE/PFPE interface, and (3) the short $(<40 \mathrm{~s})$ PFPE residence times. See Ref. 44 for details.

(44) Kasai, P. H.; Tang, W. T.; Wheeler, P. Appl. Surf. Sci. 1991, 51, 201-211. 


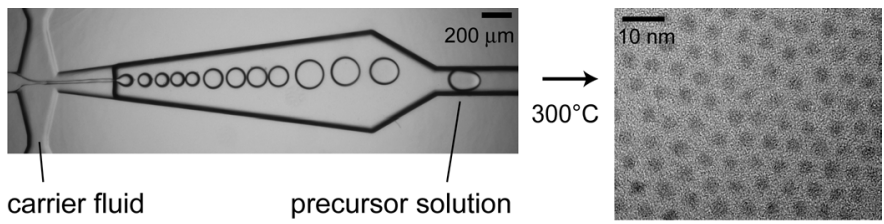

Table of Contents artwork 